\title{
Diffractive and interferometric methods to characterize photopolymers with liquid crystal molecules as holographic recording material
}

\author{
S. Gallego \\ Sergi.Gallego@ua.es
}

A. Márquez

M. Ortuño

\section{J. Francés}

\section{Pascual}

\section{A. Beléndez}

\begin{abstract}
Universidad de Alicante, Instituto Universitario de Física Aplicada a las Ciencias y las Tecnologías, Sant Vicent del Raspeig, 03690, Spain

Departamento de Física, Ingeniería de Sistemas y Teoría de la Señal. Universidad de Alicante. Apartado 99. E03080 Alicante, Spain

Universidad de Alicante, Instituto Universitario de Física Aplicada a las Ciencias y las Tecnologías, Sant Vicent del Raspeig, 03690, Spain

Departamento de Física, Ingeniería de Sistemas y Teoría de la Señal. Universidad de Alicante. Apartado 99. E03080 Alicante, Spain
\end{abstract}

Universidad de Alicante, Instituto Universitario de Física Aplicada a las Ciencias y las Tecnologías, Sant Vicent del Raspeig, 03690, Spain

Departamento de Física, Ingeniería de Sistemas y Teoría de la Señal. Universidad de Alicante. Apartado 99. E03080 Alicante, Spain

Universidad de Alicante, Instituto Universitario de Física Aplicada a las Ciencias y las Tecnologías, Sant Vicent del Raspeig, 03690, Spain

Departamento de Física, Ingeniería de Sistemas y Teoría de la Señal. Universidad de Alicante. Apartado 99. E03080 Alicante, Spain

Universidad de Alicante, Instituto Universitario de Física Aplicada a las Ciencias y las Tecnologías, Sant Vicent del Raspeig, 03690, Spain

Departamento de Óptica, Farmacología y Anatomía. Universidad de Alicante. Apartado 99. E03080 Alicante, Spain

Universidad de Alicante, Instituto Universitario de Física Aplicada a las Ciencias y las Tecnologías, Sant Vicent del Raspeig, 03690, Spain

Departamento de Física, Ingeniería de Sistemas y Teoría de la Señal. Universidad de Alicante. Apartado 99. E03080 Alicante, Spain

We present two methods, interferometry at the zero spatial frequency limit and analysis of diffracted orders for very low spatial frequency gratings, to characterize photopolymers with dispersed nematic liquid crystals. These methods provide us with real time information about the transformations taking place inside the material during recording.

[DOI: http://dx.doi.org/10.2971/jeos.2012.12024]

Keywords: diffraction and gratings, holography, holographic recording materials

\section{INTRODUCTION}

Holographic recording materials are usually characterized by recording holographic gratings [1, 2]. The main advantage of this method is that characterization and optimization of the material, and of the processes to store recorded holographic gratings, are performed simultaneously. For example, it is possible to multiplex many gratings in the material, trying to achieve high values of diffraction efficiency, and to measure the signal-to-noise ratio. Photopolymers are one of the most promising holographic recording materials. Many compositions have been developed for applications such as diffractive elements, data-storage displays, optical waveguides, etc [3][8]. However, materials with many parameters are usually involved in hologram formation, and it is difficult to determine the importance of each individual parameter in order to fit them separately.
In particular, in photopolymeric materials the interplay between polymerization and diffusion inside the material makes this task especially challenging and ambiguous. In addition there are many types of photopolymers that may be differentiated by the type of binder employed, since this component determines to a great extent the choice of monomer, dye and initiator used in the photopolymer. In the range of holographic frequencies (from 400 to 6000 lines $/ \mathrm{mm}$ ) many processes are involved in hologram formation, such as species diffusion, non-local polymerization (due to the finite size of polymer chains) [9], and shrinkage or swelling (volume changes) [10][12]. When modelling the photopolymer behaviour a wide range of spatial frequencies are recorded, from 500 lines $/ \mathrm{mm}$ to the highest material resolution [13], with different recording intensities in order to evaluate the importance of polymeriza- 
tion and monomer diffusion [14]. In recent years some authors have analzyed the diffracted order for very short exposures when recording stops [15]. Nevertheless in some works [14] the important discrepancies in the values obtained for these parameters are shown and discussed. These discrepancies result from the difficulty involved in calculating them individually.

In the field of photopolymeric materials, polymer dispersed liquid crystals, H-PDLCs, have become one of the most interesting due to their switchable design. The formation of switchable holographic gratings from polymer dispersed liquid crystals (H-PDLCs) allows switchable transmissive and reflective diffractive optics to be developed. To overcome some limitations of photopolymers and liquid crystal displays, two polymer-related technologies have been combined over the past few years with fascinating results: photopolymer holography and polymer-dispersed liquid crystals, H-PDLC $[16,17]$. Local differences in photopolymerization rates induce phase separation of discrete LC domains that occurs periodically and is commensurate with the period of the interference pattern.. When such a mixture is exposed to the optical field of two or more interfering laser beams, photopolymerization occurs more rapidly in bright than in dark regions of the interference pattern. This leads to diffusion of liquid crystalline molecules into dark regions and of monomers to bright regions. The applications, new H-PDLC compositions and optimization studies are presented in several papers [17]-[19].

We propose interferometric and diffractive methods to characterize certain properties of photopolymers as holographic recording materials, allowing the main parameters that govern diffractive image formation to be decoupled. The first method is interferometric analysis at the zero spatial frequency limit. And the second consists in recording very low spatial frequency gratings and measuring the intensity of the different diffracted orders, which permits us to fit the exact grating shape recorded and track molecular diffusion in real time. In the case analyzed in this paper, PEA photopolymers with and without dispersed liquid crystal molecules are characterized. This material is liable to contain liquid crystal particles; thus dynamic holograms or diffractive elements can be manufactured. In general, the samples are between two glass plates. This cover-plating provides mechanical support and alters surface tension (energy) effects so as to change the profile of the surface relief grating formed during exposure [14]. However, with coverplating it is not possible to measure shrinkage and swelling in real time using zero spatial frequency recording [20].

\section{EXPERIMENTAL PROCEDURE}

The first method (Figure 1) is based on an interferometer that has been successfully applied in the phase-shift versus applied voltage characterization of liquid-crystal displays (LCDs) [21]. It has good precision and, due to its quasicommon-path architecture, is a robust setup, less sensitive to changing environmental conditions and simpler to construct than Mach-Zehnder type interferometers. We used a grating with a spatial frequency of 4 lines/mm to generate a series

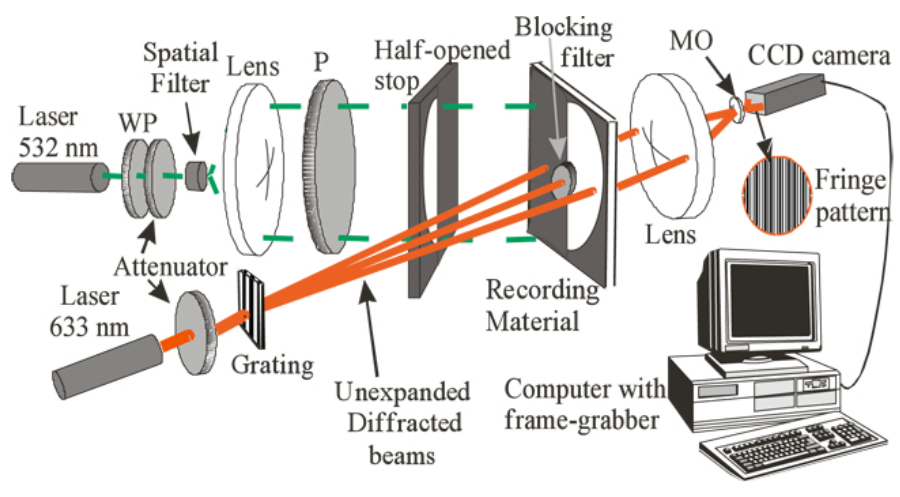

FIG. 1 Experimental setup for zero spatial frequency analysis. P is the polarizer, WP the wave plate and $\mathrm{MO}$ the microscope objective.

of diffracted orders and blocked all the orders except +1 and -1 . One of the two orders impinges on the exposed zone (illuminated by the Nd-YVO4 laser) and the other one impinges on the non-exposed zone. The distance between the two orders is approximately $1 \mathrm{~cm}$, so as to eliminate the influence of monomer diffusion in the polymerization process. Afterwards the orders are brought to interfere and from the interference pattern we can determine what happens inside the material due to polymerization (diffusion is not present). It is interesting to comment the possibility to record reflection gratings due to the Fresnel boundaries reflections. Nevertheless the reflected intensity in the last boundary between glass and air is weak in transmission geometry and in addition due to the non-local effect, the size of polymer chains, probably the recording of these reflection gratings is dramatically attenuated.

For very low spatial frequency analysis, the diffractive-based experimental setup is shown in Figure 2, where the periodic pattern is introduced using a LCD as the master which is copied onto the photopolymer. We used an expanded, collimated recording beam provided by a solid-state Verdi laser (Nd-YVO4) with a wavelength of $532 \mathrm{~nm}$ (at this wavelength the dye, yellowish eosine, exhibits maximum absorption). The periodic pattern, sinusoidal-like, is introduced by a liquid crystal display (LCD), a Sony LCD model LCX012BL, extracted from a video projector Sony VPL-V500. We used the electronics of the video projector to send the voltage to the pixels of the LCD. The LCD was used in the amplitude-mostly modulation regime by proper orientation of the external polarizers $(P)[21,22]$; then the pattern was imaged onto the material with an increased spatial frequency (a demagnifying factor of 2). Using the LCD allows us to change the period of the grating recorded in the photopolymer without moving any mechanical part of the set-up. Nevertheless, the size of the pixel, $42 \mu \mathrm{m}$, of this LCD model limits the minimum value of the spatial period in the recording material to $168 \mu \mathrm{m}$ (i.e. $8 \mathrm{LCD}$ pixels to reproduce a period). To analyze in real time the variation in efficiency of the different diffraction orders, we used an unexpanded beam of a He-Ne laser $(633 \mathrm{~nm})$ incident at a small angle with respect to the normal axis of the recording material. A diaphragm (stop2) was placed in the focal plane of the relay lens so as to eliminate the diffraction orders produced by pixelation of the LCD. The final pattern imaged onto the recording material can be expected to be low- 


\begin{tabular}{|l|l|l|l|l|l|l|l|}
\hline & C1 & C2 & C3 & C4 & C5 & C6 & C7 \\
\hline DPHPA & $2 \mathrm{~g}$ & $1.9 \mathrm{~g}$ & $1.7 \mathrm{~g}$ & $1.4 \mathrm{~g}$ & $1.5 \mathrm{~g}$ & $1.6 \mathrm{~g}$ & $1.8 \mathrm{~g}$ \\
\hline NMDETA & $0.4 \mathrm{~mL}$ & $0.4 \mathrm{~mL}$ & $0.4 \mathrm{~mL}$ & $0.4 \mathrm{~mL}$ & $0.4 \mathrm{~mL}$ & $0.4 \mathrm{~mL}$ & $0.4 \mathrm{~mL}$ \\
\hline NVP & $0.1 \mathrm{~mL}$ & $0.2 \mathrm{~mL}$ & $0.4 \mathrm{~mL}$ & $0.1 \mathrm{~mL}$ & $0.1 \mathrm{~mL}$ & $0.1 \mathrm{~mL}$ & $0.1 \mathrm{~mL}$ \\
\hline YEt & $1 \mathrm{mg}$ & $1 \mathrm{mg}$ & $1 \mathrm{mg}$ & $1 \mathrm{mg}$ & $1 \mathrm{mg}$ & $1 \mathrm{mg}$ & $1 \mathrm{mg}$ \\
\hline LC BL087 & & & & $0.6 \mathrm{ml}$ & $0.5 \mathrm{~mL}$ & $0.4 \mathrm{~mL}$ & $0.2 \mathrm{~mL}$ \\
\hline
\end{tabular}

TABLE 1 Chemical compositions analyzed.

\begin{tabular}{|l|l|l|l|l|l|l|l|l|}
\hline & C1 & C2 & C3 & C4 & C5 & C6 & C7 & n \\
\hline DPHPA & 0.7758 & 0.7326 & 0.6477 & 0.5241 & 0.5649 & 0.6061 & 0.6899 & 1.4900 \\
\hline NMDETA & 0.1792 & 0.1781 & 0.1761 & 0.1729 & 0.1739 & 0.1750 & 0.1771 & 1.4694 \\
\hline NVP & 0.0448 & 0.0891 & 0.1760 & 0.0433 & 0.0435 & 0.0437 & 0.0443 & 1.5110 \\
\hline YEt & 0.0002 & 0.0002 & 0.0002 & 0.0002 & 0.0002 & 0.0002 & 0.0002 & 1.7700 \\
\hline LC BL087 & 0 & 0 & 0 & 0.2594 & 0.2175 & 0.1750 & 0.0885 & 1.5460 \\
\hline $\mathbf{n}_{0}$ & 1.4873 & 1.4882 & 1.4900 & 1.5016 & 1.4993 & 1.4969 & 1.4922 & \\
\hline
\end{tabular}

TABLE 2 Chemical compositions analyzed.

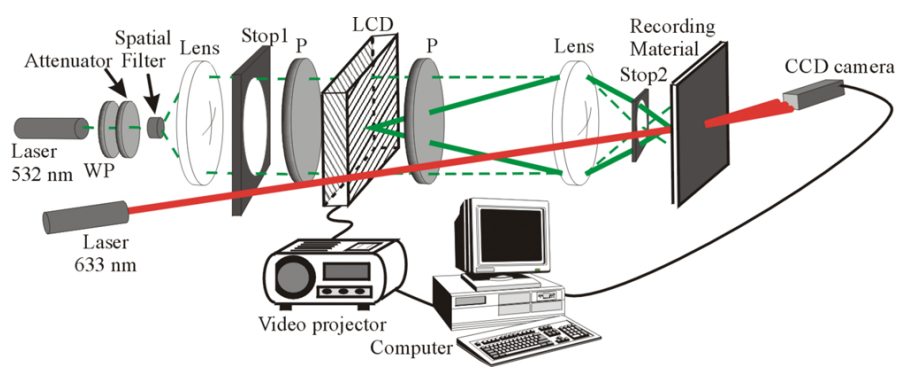

FIG. 2 Experimental set-up used to analyze the recording of gratings in real time.

pass filtered due to the finite aperture of the imaging system and especially due to the filtering process produced by stop2.

This photopolymer is composed of dipentaerythritol penta/hexa-acrylate (DPHPA) as monomer and binder, $\mathrm{N}$-vinyl pirrolidone (NVP) as crosslinker, ethyl eosin (YE) as dye and N-methyl diethanolamine (NMDETA) as radical generator. These components were mixed under red light where the material is not sensitive and at a temperature of 40 ${ }^{\circ} \mathrm{C}$ to ensure all the components, especially the crystal liquid molecules, were completely dissolved. The solution $(100 \mathrm{~mL})$ was sonicated in an ultrasonic bath, heated, mixed with a magnetic mixer and deposited between two glass plates 2 $\mathrm{mm}$ thick. The H-PDLCs layers were around $10 \mu \mathrm{m}$ thick in order to apply the electric field and to align the liquid crystal molecules, thus obtaining switchable holographic gratings. In the samples analyzed in this paper, a spacing of $106 \mu \mathrm{m}$ was used to increase the phase shift and make it possible to see the different behaviours properly, thereby reducing the error in our calculations. Our main objective was to characterize the chemical composition rather than make switchable gratings. Table 1 shows the composition of different layers analyzed at zero spatial frequencies. These compositions were designed in such a way as to keep the liquid volume constant. In the first three compositions we analyzed the influence of the crosslinker concentration, and in the last four the influence of the liquid crystals in the polymerization process.

Once the volume fractions are introduced it is easy to obtain the volume fraction for each component, $\phi^{(i)}$. Using the values of refractive index, $n_{i}$, of each compound together with the Lorentz-Lorenz equation [23], the refractive index of the layer before recording, $n_{0}$, can be calculated

$$
\frac{n_{0}^{2}-1}{n_{0}^{2}+2}=\sum_{i=1}^{k} \frac{n_{i}^{2}-1}{n_{i}^{2}+2} \phi^{(i)}
$$

These values were checked using Abbe refractometer (Model AYA-15) measurements and are presented in Table 2. As can be deduced from Table 2 (the refractive index of LC is the averaged one in the random orientation), in compositions C4 and C5 the liquid crystal volume fraction is higher than $20 \%$. This can prevent huge variations in the refractive index at zero spatial frequency recording.

\section{RESULTS AND DISCUSSION}

As mentioned in the introduction, parameters have classically been fitted based on a combined study of the evolution of diffracted efficiency during the recording of holographic gratings and the angular responses of the recorded gratings [24]. In this section we present the alternative characterization proposed. The goal is to understand what happens in the material so as to be able to fit directly all the parameters and model any application of the material. Therefore, we first analyze the material behaviour for each composition in the absence of diffusion. In this sense we analyze the influence of crosslinker and liquid crystal concentrations. In particular we study how the presence of liquid crystals affects the changes in refractive index. In the last section of results we introduce the method for calculating the diffusion of molecules inside the material.

\subsection{Zero spatial frequency limit}

In this study we analyzed different chemical compositions of PEA photopolymers at the zero spatial frequency limit. We also studied the influence of the dispersed liquid crystal molecules in the polymerization process.

We could only measure the results in transmission, where 
both refractive index and volume variations have an influence. The phase shift measured in the case of transmission $\left(P S_{t}\right)$ can be described as follows:

$$
P S_{t}=\frac{2 \pi}{\lambda} \frac{d(t) \cdot \Delta n}{\cos \alpha^{\prime}}-P S_{S h r}
$$

where $d(\mathrm{t})$ is the new "solid" layer thickness [20]-[25], $\alpha$ ' is the angle inside the material and $P S_{S h r}$ is the phase shift due to shrinkage. For cover-plated photopolymers we can consider that the volume between the two glass substrates remains constant.

The equation can be simplified for small incident angles $\left(\cos \alpha^{\prime}=\cos \alpha=1\right)$ and used to calculate the refractive index variation. In this case, for the whole layer the refractive index variation $\left(\Delta n_{w}\right)$ can be calculated as follows:

$$
\Delta n_{w}=\frac{\lambda P S_{t} \cos \alpha^{\prime}}{2 \pi d_{0}}
$$

Throughout the polymerization process, the volume between the glass plates remains constant.

$$
\sum_{i=1}^{k} \phi^{(i)}=1
$$

where $\phi^{(i)}$, are the volume fractions of each component, and the volume fraction of the holes, $\phi^{(h)}$, can also be considered. While the collapse of holes will result in a reduction in the overall volume, the total volume fraction is by definition conserved. We can assume that $\phi^{(h)}$, and $\phi^{(C L)}$ (volume fraction of the liquid crystals) remain constant during exposure at the zero spatial frequency limit. Therefore we can assume that:

$$
\Delta \phi^{(D P H P A)}+\Delta \phi^{(N M D E T A)}+\Delta \phi^{(N V P)}+\Delta \phi^{(Y E t)}=\phi^{(p)}
$$

Where, $\Delta \phi^{(i)}$ is the variation in each volume fraction during polymerization and $\phi^{(p)}$ the polymer volume fraction.

At the zero spatial frequency limit, when diffusion does not take place, the decrease in monomer concentration depends on the recording intensity, $I$, the dependency of the polymerization and intensity, $\gamma$, initial monomer concentration and the polymerization reaction constant, $K_{R}$.

$$
\phi^{(m)}(t)=\phi_{0}^{(m)} e^{-I^{\gamma} K_{R} t}
$$

The model used in this paper is simple and based on the classical model proposed by Zhao et al. [26]. The aim of the paper is to propose and demonstrate a simple diffractive and interferometric method to calculate the first approximation of the polymerization rate and monomer diffusion. It is important to note that the gradient of monomer concentration in depth due to the dye absorption analyzed in previous works $[27,28]$ has been disregarded in this first approximation to the problem. In this sense it is important to mention the contributions of Sheridan et al. to clarify all the parameters involved in the polymerization process such as the influence of oxygen inhibiting [29], the effects of the dye kinetics, and polymer chain initiation, propagation and termination [30]-[32] that can be studied using real zero analysis in future works. In any case the parameters obtained using the presented methodology produce good results when introduced in simple models as it is shown in references $[33,34]$

Once the first assumption is made, following the same steps presented in $[20,25]$ we can obtain the polymerization rate as a function of the experimental values of the phase shift, PS, during recording.

$$
\ln \left(1-\frac{P S(t)}{P S_{\infty}}\right)=-I^{\gamma} K_{R} t=-F_{R} t
$$

Furthermore if the quantities of the different compounds are known, it is possible to obtain the polymer refractive index using the Lorentz-Lorenz equation [23]. To make the calculus more accurate, it is possible to know the exact concentration of each component at the end of zero frequency recording using additional methods such as interferometry. In any case, interesting information can be extracted solving the following equation for some particular cases:

$$
\frac{n_{p}^{2}-1}{n_{p}^{2}+2}=\frac{1}{\phi_{f}^{(p)}}\left[\frac{n_{f}^{2}-1}{n_{f}^{2}+2}-\sum \frac{n_{i}^{2}-1}{n_{i}^{2}+2} \phi_{f}^{(i)}\right]
$$

Where $n_{f}$ is the final refractive index of the layer, $n_{p}$ is the refractive index of the polymer formed during exposure, and $\phi_{f}^{(i)}$ is the final volume fraction for compound $i$. In the cases analyzed in this paper, due to the high value of dye concentration, the samples exhibit high absorption, (the transmittances are around $1 \%$ ). In addition at the end of the exposing process we detected the presence of very little dye. Thus we assume that in the exposed zones all the components, except the liquid crystals, are polymerized. With this assumption equation 8 can be reduced to:

$$
\frac{n_{p}^{2}-1}{n_{p}^{2}+2}=\frac{1}{\phi_{f}^{(p)}}\left[\frac{n_{f}^{2}-1}{n_{f}^{2}+2}-\frac{n_{L C}^{2}-1}{n_{L C}^{2}+2} \phi_{f}^{(L C)}\right]
$$

Where $n_{L C}$ is the average refractive index of the liquid crystal used.

After presenting some of the information that it is possible to extract from zero spatial frequency recording, it is interesting to analyze the behavior of the first three compositions in order to study the effect of NVP as crosslinker in the photopolymer. The results are depicted in Figure 3. As in the case of higher NVP values, the curve can be seen to grow faster initially; however the saturation phase shift is similar. This effect can be explained by the Lorentz-Lorenz equation. When the crosslinker concentration increases, the polymer refractive index also increases. But due to the high refractive index of NVP in comparison with the other compounds, when it is consumed the average refractive index of the illuminated area decreases too. The values obtained using Eq. (9) for compositions 1,2 and 3 are 1.496392, 1.497334 and 1.499188 respectively. It can thus be seen that multiplying the NVP concentration by a factor of 4 has very little effect on the polymer generated.

Following the steps described previously and using Eq. (7) it is possible to obtain the polymerization rate of $\mathrm{C} 1$. As can be seen in this figure the correlation parameter indicates that there is agreement between experimental data and theoretical behaviour. 8 . The value at $t=0$ in the linear fitting should be 0 , 


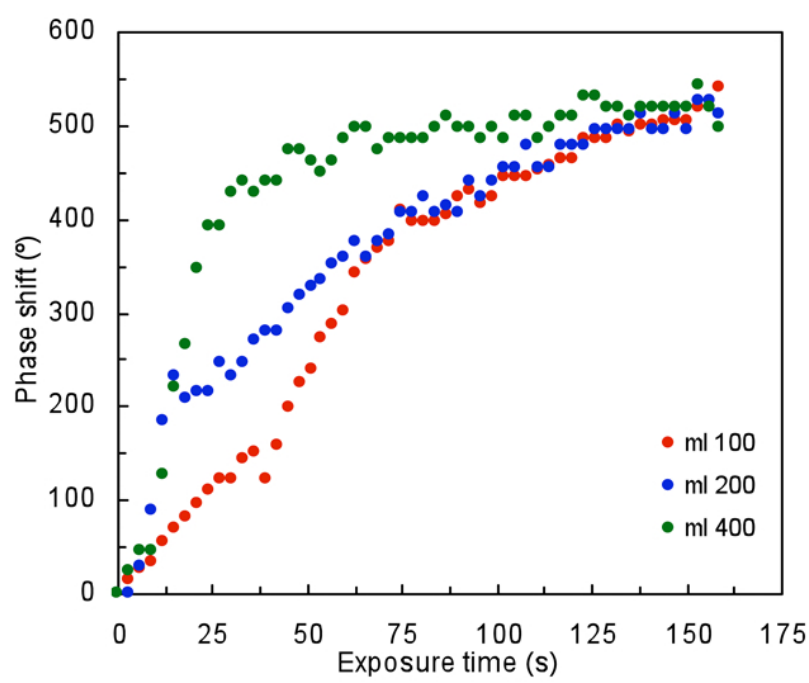

FIC. 3 Behavior of Photopolymers $C_{1}, C_{2}$ and $C_{3}$ at the zero spatial frequency limit in transmission.

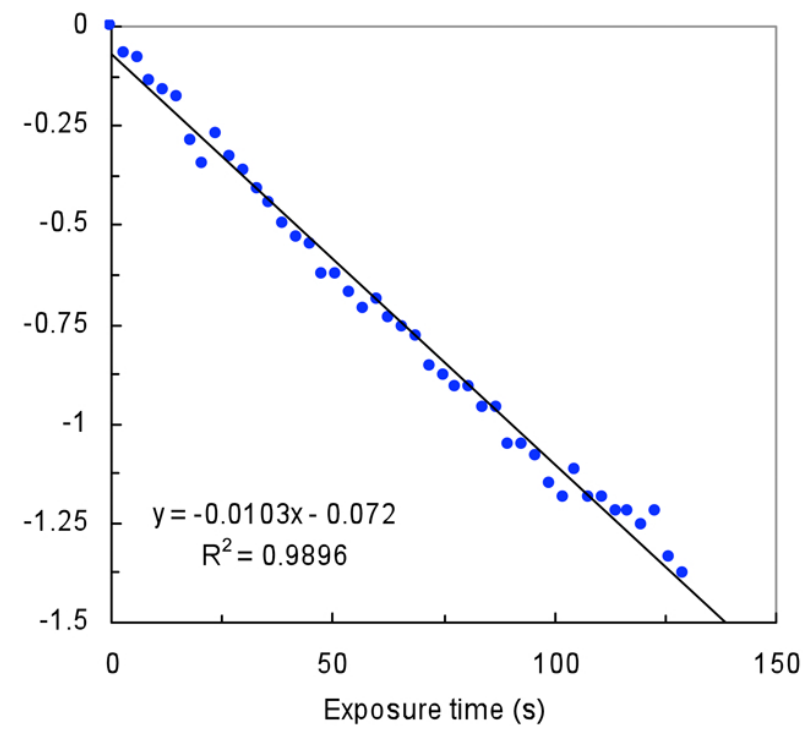

FIG. 4 Fitting of the polymerization rate for polymer $\mathrm{C}_{1}$ at the zero spatial frequency limit.

but it is well known that the Trommsdorff effect explains how the increase of viscosity reduces the polymerization rate [35], as can be seen in the last points of the Figure 4 .

In order to obtain information about the effect of incorporating LC in the photopolymerizable solution using real zero frequency recording, the phase shift between illuminated and non-illuminated zones for compositions 1, 4, 5, 6 and 7 is shown in Figure 5. As can be seen in this figure, the presence of LC molecules produces a decrease in phase shift. The values obtained for C4, C5, C6 and C7 using Eq. (9) are 1.491759, $1.491864,1.494553$ and 1.495925 respectively. Therefore we can conclude that incorporation of LC molecules results in only a small change in polymer refractive index. As zero spatial frequency recording prevents diffusion of liquid crystalline molecules into dark regions and of monomers to bright regions, the uniform concentration of LC throughout the whole layer makes it difficult for the refractive index to change.

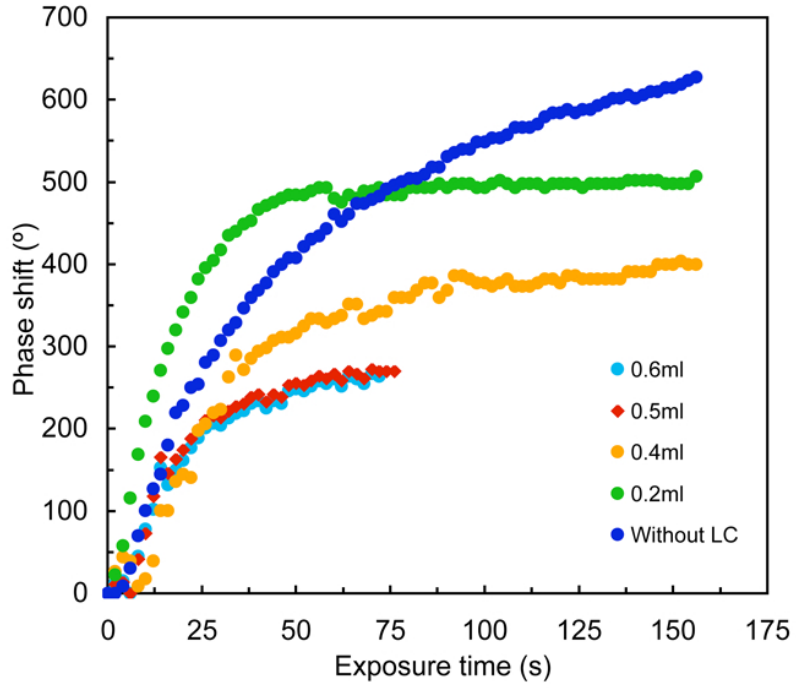

FIC. 5 Behavior of Photopolymers $\mathrm{C}_{1}, \mathrm{C}_{4}, \mathrm{C}_{5}, \mathrm{C}_{6}$ and $\mathrm{C}_{7}$ at the zero spatial frequency limit in transmission.

\subsection{Very low spatial frequency recording}

After demonstrating the utility of zero spatial frequency recording for studying the influence of some variations in the material composition when diffusion does not take place, it is interesting to use very low spatial frequency recording to analyze in real time the influence of species diffusion in photopolymers. In the hologram formation analysis, the diffusion times are very short, less than $0.1 \mathrm{~s}$, and it is impossible to measure them. Therefore, for spatial frequencies around 1000 lines/mm, species diffusion can only be determined indirectly. For very low spatial frequency recording we can measure more than 8 diffracted orders and obtain reliable information about the phase profile recorded in the material and its evolution after recording due to diffusion.

After measuring the diffracted efficiency of the main orders as a function of time we used the Fermi-Dirac function to obtain the recorded profile [22]. The Fermi-Dirac based profile is a better option than the super-Gaussian based profile used in previous studies [36] for two main reasons: the parameters in the Fermi-Dirac function are continuous, whereas in the super-Gaussian function the parameter describing the sharpness of the edges is discrete, thus there is no gradual transition between the fitted profiles. Furthermore, the Fermi-Dirac function provides a very good fit to the cosine function, which may be considered a reasonable limiting case when edges become highly smoothed by diffusion. The mathematical expression for the amplitude transmission $t(x)$ of a transparent phase element showing a Fermi-Dirac phase profile $\varphi(x)$ can be written as follows,

$$
\begin{gathered}
t(x)=\exp (i \varphi(x)) \\
\varphi(x)=\varphi_{0}\left(1+\exp \left(\alpha \cdot\left(\frac{|x|}{\Omega}-1\right)\right)\right)^{-1}
\end{gathered}
$$

where $\varphi_{0}$ is the asymptotic phase modulation depth (for an $\mathrm{x}$ interval $(-\infty,+\infty)), \Omega$ controls the symmetry (or duty cycle) of the profile, and $\alpha$ is the parameter characterizing the function shape and sharpness of the edges. The function is limited to 


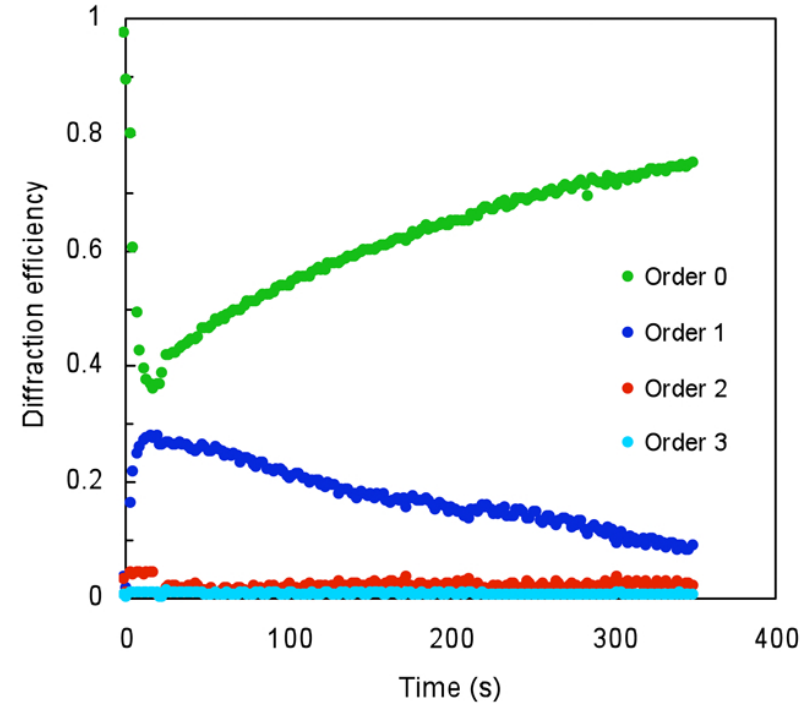

FIG. 6 Grating with spatial period of $0.168 \mathrm{~mm}$, diffraction intensities of the first 4 orders.

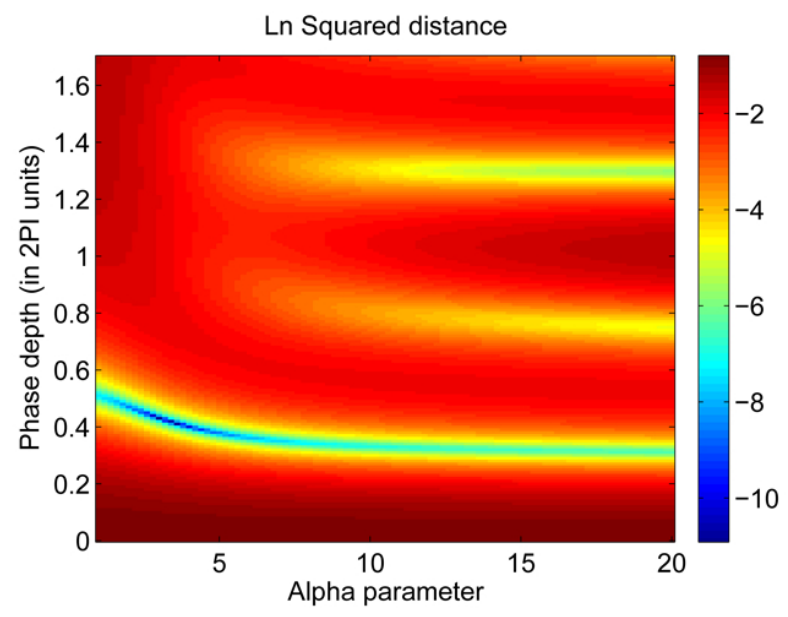

FIG. 7 Search for the optimum phase profile after $16 \mathrm{~s}$ of exposure.

an x-interval $(-1,1)$, and repeated periodically. We considered symmetric gratings, which for this $\mathrm{x}$-interval corresponds to the value $\Omega=0.5$. We rescaled the phase values obtained in the $\mathrm{x}$-interval $(-1,1)$ so that the phase modulation depth in this finite interval equals the asymptotic phase modulation depth [22].

We compared the diffraction efficiencies calculated numerically using the Fermi-Dirac model with the experimental values for the first 9 orders (orders $0,1, .$. and 8 ).

In order to demonstrate the utility of the proposed method we recorded a sinusoidal grating with a spatial period of $168 \mu \mathrm{m}$ for $16 \mathrm{~s}$ using the material C7. After recording we continued measuring the intensity of the diffracted orders so as to analyze the effects of species diffusion on the diffractive element recorded. The results are shown in Figure 6. As can be seen the diffraction efficiency of order 1 achieves a diffraction efficiency of $28 \%$ just when the recording stops and decreases after exposure. When the grating remains under red illumination all the energy goes back to the order 0 due to diffusion.

Once we stored the intensities of different diffracted orders as

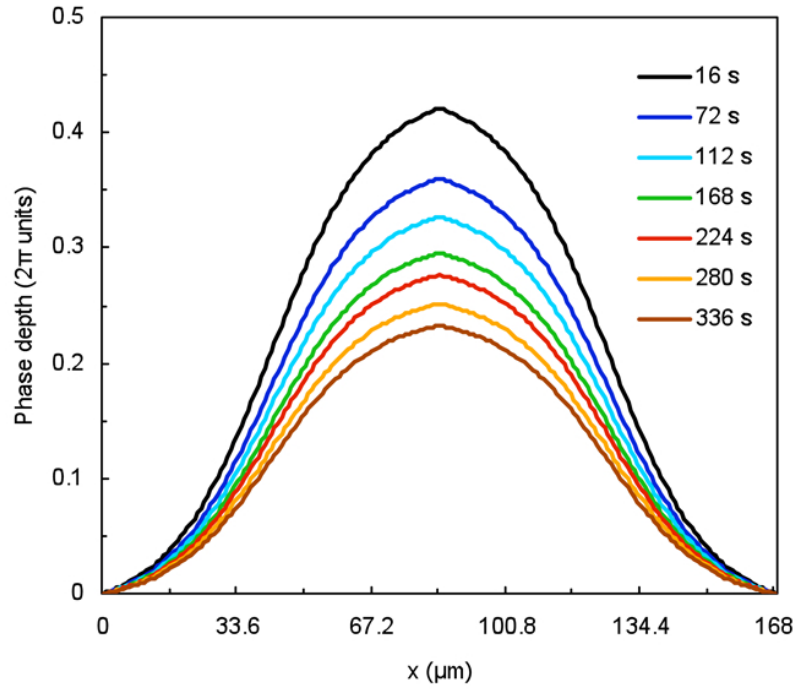

FIG. 8 Estimated profiles for different times (16, 72, 112, 168, 224, 280 and 336s). Exposure stops at $16 \mathrm{~s}$ (the absolute value for phase depth is shown).

a function of time, we fitted the experimental data to find the best phase profile. The validity of the fittings around the phase depth value considered as physically reasonable is shown in Figure 7, where the local minimum is clearly detected.

Using this method we fitted some profiles to analyze the phase grating evolution once the exposure is finished. The points analyzed are represented in Figure 8. In this figure the phase modulation can be seen to decrease due to species diffusion.

The Fermi-Dirac fittings provide important information, which can be used to calculate the average species diffusion inside the material [22, 37]. In this sense, to solve Fick's law (Eq. (12)) after a periodic illumination we proposed the following model. Due to exposure a species concentration distribution is generated inside the photopolymer $\left(\phi^{(i)}\right)$. Exponential decay (increase) of species concentration in the non-exposed (exposed) zones due to species diffusion begins when exposure stops to achieve the average value of the residual specie concentration $\left(\phi_{f}^{(i)}\right)$, corresponding to the point when monomer diffusion eventually stops due to uniform monomer distribution. It is important to note that in the case of the PEA photopolymer analyzed, there are many species diffusing after illumination, such as DPHPA, NVP, etc. Therefore using this method we obtain the average diffusion value for all the molecules, $\phi^{(a v g)}$.

$$
\frac{\partial \phi^{(i)}(x, t)}{\partial t}=\frac{\partial}{\partial x}\left[D(x, t) \frac{\partial \phi^{(i)}(x, t)}{\partial x}\right]
$$

We can thus assume the species concentration after exposure as:

$$
\phi^{(i)}(x, t)=\phi_{f}^{(i)}+\Delta \phi^{(i)}(x) \exp \left(\frac{-t}{\tau}\right)
$$

where the species modulation is $\Delta \phi^{(i)}(x)$ and $\tau$ is the characteristic time of monomer variation and can be calculated by fitting the phase depth of the grating variation with time $[22,37]$. When we introduce this expression in Eq. (12) we obtain:

$$
\Delta \phi^{(i)}(x, t)=-\tau \frac{\partial}{\partial x}\left[D(x, t) \frac{d \Delta \phi^{(i)}(x)}{d x}\right]
$$


In a first approximation we can assume a sinusoidal distribution of the modulation of species concentrations and for the illumination $(I)$ :

$$
\begin{gathered}
\Delta \phi^{(i)}(x)=\Delta \phi_{0}^{(i)}(x)_{0} \cos \left(\frac{2 \pi x}{\Lambda}\right) \\
I(x)=I_{0}\left(1-\cos \left(\frac{2 \pi x}{\Lambda}\right)\right)
\end{gathered}
$$

where $\Lambda$ is the grating period. This assumption only makes sense just for very short exposures and sinusoidal distribution of the recording intensity. Therefore the Eq. (14) can be rewritten as:

$$
\operatorname{tg}\left(\frac{2 \pi}{\Lambda}\right) \frac{\partial}{\partial x} D(x, t)+\frac{2 \pi}{\Lambda} D(x, t)-\frac{1}{2 \pi \tau}=0
$$

This gives a first order inhomogeneous differential equation with constant coefficients; therefore, the general solution is:

$$
D(x)=\frac{\Lambda^{2}}{4 \pi^{2} \tau}+D_{1} \csc \left(\frac{2 \pi}{\Lambda} x\right)
$$

It is important to note the simplicity of Eq. (14) - only two parameters determine the value of $D: \Lambda$ and $\tau$. In the case analyzed in this section the exposure time is $16 \mathrm{~s}$ and the exposure intensity is $1 \mathrm{~mW} / \mathrm{cm}^{2}$. Therefore the polymer concentration is small and we can assume that $D$ is spatially independent.

$$
D=\frac{\Lambda^{2}}{4 \pi^{2} \tau}
$$

In our analysis of the evolution of refractive index distribution in time, we noted that the phase modulation, $\Delta p h$, becomes time independent after a time range of several minutes to a few days after exposure. The approximation to this value is exponential. Next we show how we can obtain the value for the parameter $\tau$, needed in Eq. (19), from the fitting of the temporal variation for $\Delta p h$. If we use Eq. (13) we can obtain:

$$
\Delta p h(x, t)=V_{m} \Delta \phi^{(a v g)}(x)\left(\exp \left(\frac{-t}{\tau}\right)-1\right)
$$

This value tends asymptotically (in practice in some minutes) to the value:

$$
\Delta p h(x, t \rightarrow \infty)=-V_{m} \Delta \phi^{(a v g)}(x)
$$

Thus, Eq. (15) can be rewritten as:

$$
\begin{aligned}
& \Delta p h(x, t \rightarrow \infty)-\Delta p h(x, t) \\
= & \Delta p h(x, t \rightarrow \infty) \exp (-t / \tau)
\end{aligned}
$$

If we apply the logarithm to both sides of the expression, we obtain:

$$
\begin{aligned}
& \ln (\Delta p h(x, t \rightarrow \infty)-\Delta p h(x, t)) \\
= & \ln (\Delta p h(x, t \rightarrow \infty))^{-t} / \tau
\end{aligned}
$$

It should be noted that we did not assume any specific profile for $\Delta p h(x)$ to derive Eq. (18). The movement of species, except LC, from the dark zones to bright ones creates refractive index variations in the dark zones and in the bright ones.

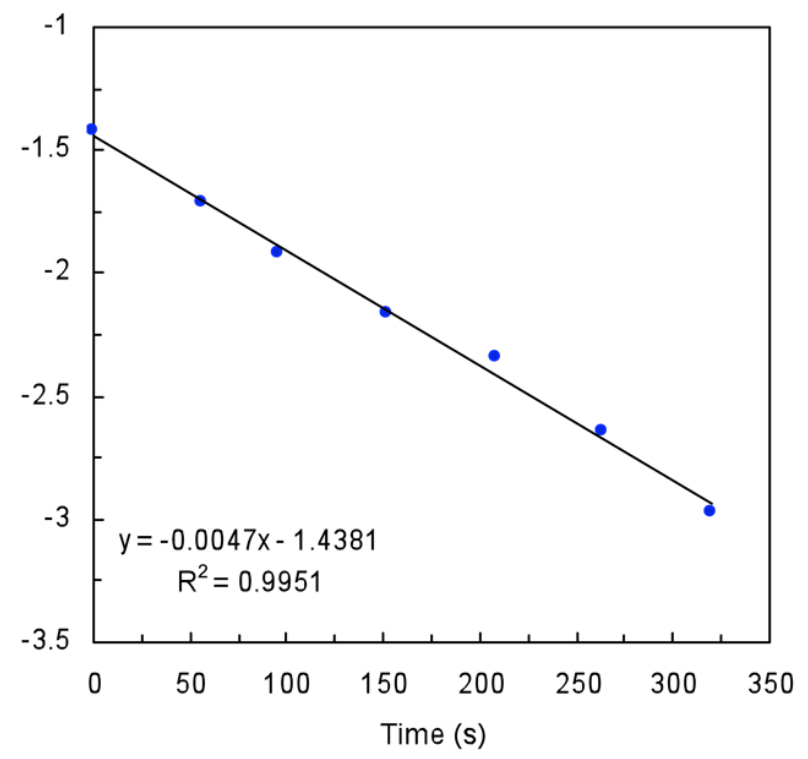

FIG. 9 Logarithm of variation of phase depth as a function of time for a grating with a spatial period of $0.168 \mathrm{~mm}$.

In order to determine the value of $\tau$ we applied Eq. (18) at $x=0$, where illumination takes the maximum value. We studied the evolution of the diffraction efficiency for 4 days in order to estimate an accurate value of the limit of $\Delta n(t \rightarrow \infty)$. In Figure 9 we represent the fitting used to calculate $\tau$. The value of $R^{2}$ represented in this figure shows the agreement between the experiments and the theoretical behavior assumed. Using the data from Figure 9 we obtain the following values: $\tau=213 \mathrm{~s}$ and $D=3,36 \cdot 10^{-8} \mathrm{~cm}^{2} \mathrm{~s}^{-1}$. This value of $D$ is three times higher than that obtained in acrylamide materials [37] and more than ten times higher than that obtained in sodium acrylate materials [25]. The high velocities of $D$ obtained make sense because the photopolymers analyzed in this paper are liquid at ambient conditions, the exposure times are short (16s) and the polymer concentration in the exposed zones is low.

\section{CONCLUSION}

Using interferometric and diffractive analysis we characterized a photopolymer with and without liquid crystal molecules. Combining the two methods we were able to analyze polymerization and diffusion separately and thus discuss the influence of the different components such as liquid crystal molecules, crosslinker, etc. on grating formation. We also presented some simply models using interferometric methods to fit the polymerization rate and average species diffusion inside the material. The interferometric and diffractive analyses have been applied to calculate the first approximation of the main parameters for different composition of H-PDLCs photopolymer. The results of the fittings remark the importance of these methods to analyze the photopolymers as holographic recording material. 


\section{ACKN OWLEDGEMENTS}

This work was supported by the Ministerio de Ciencia e Innovación of Spain under projects FIS2011-29803-C02-01 and FIS2011-29803-C02-02 and by the "Generalitat Valenciana" of Spain (projects PROMETEO/2011/021 and ISIC/2012/013).

\section{References}

[1] S. Martin, P. E. Leclere, Y. L. M. Renotte, V. Toal, and Y. F. Lion, "Characterization of an acrylamide-based dry photopolymer holographic recording material," Opt. Eng. 33, 3942 (1994).

[2] K. Curtis, and D. Psaltis, "Characterization of the Du-Pont photopolymer for 3-dimensional holographic storage," Appl. Optics 33(23), 5396-5399, (1994).

[3] T. J. Trout, J. J. Schmieg, W. J. Gambogi, and A. M. Weber, "Optical photopolymers: design and applications," Adv. Mater. 10, 1219-1224 (1998).

[4] V. A. Barachevskii, "Photopolymerizable recording media for threedimensional holographic optical memory," High. Energy Chem. 40, 131-141 (2006).

[5] W. J. Gambogi, A. M. Weber, and T. J. Trout, "Advances and applications of DuPont holographic photopolymers," SPIE 2043, 2-13 (1993).

[6] H.-Y. S. Li, and D. Psaltis, "Three-dimensional holographic disks," Appl. Optics 33, 3764-3774 (1994).

[7] S. Orlic, S. Ulm, and H. J. Eichler, " $3 \mathrm{D}$ bit-oriented optical storage in photopolymers," J. Opt. A 3, 72-81 (2001).

[8] Márquez, C. Neipp, S. Gallego, M. Ortuño, A. Beléndez and I. Pascual, "Edge enhanced imaging using PVA/acrylamide photopolymer gratings," Opt. Lett. 28, 1510-1512 (2003).

[9] J. T. Sheridan, and J. R. Lawrence, "Nonlocal-response diffusion model of holographic recording in photopolymer," J. Opt. Soc. Am. A 17, 1008-1014 (2000).

[10] K. Pavani, I. Naydenova, S. Martin, V. Toal, "Photoinduced surface relief studies in an acrylamide-based photopolymer," J. Opt. A-Pure Appl. 0pt. 9, 43-48 (2007).

[11] I. Naydenova, E. Mihaylova, S. Martin, and V. Toal, "Holographic patterning of acrylamide-based photopolymer surface," Opt. Express $13,4878-4889$ (2005).

[12] S. Gallego, A. Márquez, M. Ortuño, J. Francés, S. Marini, A. Beléndez, and I. Pascual, "Surface relief model for photopolymers without cover plating," Opt. Express 19, 10896-10906 (2011).

[13] J. T. Sheridan, M. Downey, and F. T. O'Neill, "Diffusion based model of holographic grating formation in photopolymers: generalized non-local material responses," J. Opt. A-Pure Appl. Opt. 3, 477-488 (2001).

[14] C. E. Close, M. R. Gleeson, and J. T. Sheridan, “Monomer Diffusion Rates in Photopolymer Material: Part I: Low Spatial Frequency Holographic Gratings," J. Opt. Soc. Am. B 28, 658-666 (2011).

[15] J. V. Kelly, M. R. Gleeson, C. E. Close, F. T. 0' Neill, J. T. Sheridan, S. Gallego, and C. Neipp, "Temporal analysis of grating formation in photopolymer using the nonlocal polymerization-driven diffusion model," Opt. Express 13, 6990-7004 (2005).

[16] N. Suzuki, Y. Tomita, K. Ohmori, M. Hidaka, and K. Chikama, "Highly transparent $\mathrm{ZrO}_{2}$ nanoparticle-dispersed acrylate photopolymers for volume holographic recording," Opt. Express 14, 12712-12719 (2006).
[17] S. Harbour, J. V. Kelly , T. Galstian, and J. T. Sheridan, "Optical birefringence and anisotropic scattering in acrylate based holographic polymer dispersed liquid crystals," Opt. Commun. 278, 28-33 (2007).

[18] K. R. Sun, Y. S. Kang, and B. K. Kim, "Transflective multiplexing of holographic polymer dispersed liquid crystal using Si additives," Polymer Letters 5, 73-81 (2010).

[19] T. J. Bunning, L. V. Natarajan, V. P. Tondiglia, and R. L. Sutherland, "Holographic polymer-dispersed liquid crystals (H-PDLCS)," Annu. Rev. Mater. Sci. 30, 83-115 (2000).

[20] S. Gallego, A. Márquez, D. Méndez, M. Ortuño, C. Neipp, M. L. Alvarez, A. Beléndez, E. Fernández, and I. Pascual, “Analysis of PVA/AA based photopolymers at the zero spatial frequency limit using interferometric methods," Appl. Optics 47, 2556-2563 (2008).

[21] A. J. Bergeron, F. Gauvin, D. Gagnon, H. Gingras, H. H. Arsenault, and $M$. Doucet, "Phase calibration and applications of a liquid crystal spatial light modulator," Appl. Optics 34, 5133-5139 (1995).

[22] S. Gallego, A. Márquez, D. Méndez, S. Marini, A. Beléndez, and I. Pascual, "Spatial-phase-modulation-based study of polyvinylalcohol/acrylamide photopolymers in the low spatial frequency range," Appl. Optics 48, 4403-4413 (2009).

[23] M. Born, and E. Wolf, Principles of Optics (Pergamon Press. Oxford, 1980).

[24] M. Ortuño, E. Fernández, S. Gallego, A. Beléndez, and I. Pascual, "New photopolymer holographic recording material with sustainable design," Opt. Express 15, 12425 (2007).

[25] S. Gallego, A. Márquez, M. Ortuño, S. Marini, and J. Francés, "High environmental compatibility photopolymers compared to PVA/AA based materials at zero spatial frequency limit," Opt. Mater. 33, 531-537 (2010).

[26] G. Zhao, and P. Mouroulis, "Extension of a diffusion model for holographic photopolymers," J. Mod. Opt. 42, 2571-2573 (1995).

[27] S. Gallego, C. Neipp, M. Ortuño, A. Benléndez, E. Fernández, and I. Pascual, "Analysis of monomer diffusion in depth in photopolymer materials," Opt. Commun. 274, 43-49 (2007).

[28] S. Gallego, M. Ortuño, C. Neipp, A. Márquez, A. Beléndez, E. Fernández, and I. Pascual, "3-dimensional characterization of thick grating formation in PVA/AA based photopolymer," Opt. Express 14, 5121-5128 (2006).

[29] M. R. Gleeson, J. V. Kelly, C. E. Close, F. T. O'Neill, and J. T. Sheridan, "Effects of absorption and inhibition during grating formation in photopolymer materials," J. Opt. Soc. Am. B 23, 2079-2088 (2006).

[30] S. Liu, M. R. Gleeson, J. Guo, and J. T. Sheridan, "Optical characterization of photopolymers materials: Theoretical and experimental examination of primary radical generation," Appl. Phys. B 100, 559-569 (2010).

[31] D. Sabol, M. R. Gleeson, S. Liu, and J. T. Sheridan, "Photoinitiation study of Irgacure 784 in an epoxy resin photopolymer," J. Appl. Phys. 107, 053113 (2010).

[32] S. Liu, M. R. Gleeson, J. Guo, and J. T. Sheridan, "High intensity response of photopolymer materials for holographic grating formations," Macromolecules 43, 9462-9472 (2010).

[33] S. Gallego, A. Márquez, M. Ortuño, S. Marini, I. Pascual, and A. Beléndez, "Monomer diffusion in sustainable photopolymers for diffractive optics applications," Opt. Mater. 33, 1626-1629 (2011).

[34] S. Gallego, A. Márquez, M. Ortuño, J. Francés, I. Pascual, and A. Beléndez, "Relief diffracted elements recorded on absorbent photopolymers," Opt. Express 20, 11218-11231 (2012). 
[35] J. V. Kelly, F. T. O’Neill, J. T. Sheridan, C. Neipp, S. Gallego, and M. Ortuño, "Holographic photopolymer materials: non-local polymerisation driven diffusion under non-ideal kinetic conditions," J. Opt. Soc. Am. B 22, 407-416 (2005).

[36] A. Márquez, J. Campos, M. J. Yzuel, I. Pascual, A. Fimia, and A. Beléndez, "Production of computer-generated phase holograms using graphic devices: application to correlation filters," opt. Eng. 39, 1612-1619 (2000).
[37] S. Gallego, A. Márquez, S. Marini, E. Fernández, M. Ortuño, and I. Pascual, "In dark analysis of PVA/AA materials at very low spatial frequencies: phase modulation evolution and diffusion estimation," Opt. Express 17, 18279-18291 (2009). 